\title{
Análisis de imágenes satelitales Landsat 8 por componentes principales y MNF para la detección de zonas del derrame de petróleo Repsol en la Costa Peruana
}

Analysis of Landsat 8 satellite images by main components and MNF for the detection of areas of the Repsol oil spill on the Peruvian Coast

Análise de imagens de satélite Landsat 8 por componentes principais e MNF para a detecção de áreas do derramamento de óleo Repsol na costa peruana

\section{ARTÍCULO GENERAL}

Jaime Yelsin Rosales Malpartida jrosalesm@uni.pe

https://orcid.org/0000-0003-4574-5172

Universidad Nacional de Ingeniería, Lima, Perú
Hebbert Alexander Apaza Mamani

hebbert.apaza.m@uni.pe

https://orcid.org/0000-0001-9094-4563

Universidad Nacional de Ingeniería, Lima, Perú

Recibido 02 de Octubre 2021 | Arbitrado y aceptado 14 de Diciembre 2021 | Publicado en 04 Marzo 2022

\section{RESUMEN}

La contaminación por el derrame de petróleo en la costa peruana causada por el buque de bandera italiana Mare Doricum, propiedad de la empresa española Repsol no solo afectó gravemente a la flora y fauna marina sino también a cientos de familias de pescadores peruanos dejándolos sin trabajo. por ello la vigilancia de los efectos de estos incidentes es muy importante para la salud pública y el cuidado del medio ambiente. Las misiones satelitales son una herramienta muy eficiente para identificar contaminantes como los derrames de petróleo. Este estudio tiene como objetivo detectar el área del derrame de petróleo utilizando el sensor multiespectral Landsat 8. Para detectar el derrame de petróleo con mayor precisión se realizó diversos preprocesamientos a la imagen satelital Landsat 8 como la conversión a reflectancia, normalización con el método Bandmax-min de la imagen de la zona afectada y finalmente se realizó los métodos de análisis de componentes principales (ACP) y la Fracción de Mínimo Ruido (MNF por sus siglas en inglés). Todos los resultados fueron analizados mediante la programación del software libre $\mathrm{R}$, dichos resultados experimentales indicaron claramente que el método MNF es el más adecuado para detectar el derrame de petróleo con mayor precisión a través de imágenes multiespectrales Landsat 8.

Palabras clave: Landsat 8 , derrame de petróleo, Repsol, normalización, procesamiento de imágenes, ACP y MNF.

\section{ABSTRACT}

Pollution by oil spill on the Peruvian coast caused by the Italian flagship Mare Doricum, owned by the Spanish company Repsol not only seriously affected Flora and Fauna Marina but also hundreds of Peruvian fishermen families leaving them without work. therefore, monitoring the effects of these incidents is very important for public health and care for the environment. Satellite missions are a very efficient tool to identify pollutants such as oil spills. This study aims to detect the oil spill area using the Multispectral Landsat sensor 8. To detect the oil spill more precisely, various preprocessing was performed on the Landsat Satellite image as the conversion to reflectance, standardization with the BANDMAX method. min of the image of the affected area and finally the principal component analysis (ACP) and the Minimum Noise Fraction (MNF) methods were performed. All results were analyzed by programming free Software $R$, such experimental results clearly indicated that the MNF method is the most suitable for detecting the oil spill more accurately through multispectral Landsat 8 images.

Keywords: Landsat 8, oil spill, Repsol, normalization, image processing, $\mathrm{ACP}$ and MNF.

\section{RESUMO}

A poluição causada pelo derramamento de óleo na costa peruana causado pelo navio de bandeira italiana Mare Doricum, propriedade da empresa espanhola Repsol, afetou gravemente a flora e a fauna marinha, mas também centenas de famílias de pescadores peruanos, deixando-os sem trabalhar. portanto, monitorar os efeitos desses incidentes é muito importante para a saúde pública e o cuidado com o meio ambiente. As missões de satélite são uma ferramenta muito eficiente para identificar poluentes como derramamentos de óleo. $\mathrm{O}$ objetivo deste estudo é detectar a área do derramamento de óleo utilizando o sensor multiespectral Landsat 8. Para detectar o derramamento de óleo com maior precisão, diversos pré-processamentos foram realizados na imagem do satélite Landsat 8 , como conversão para refletância, foram realizados a normalização com o Bandmaxmin da imagem da área afetada e por fim os métodos de análise de componentes principais (ACP) e da fração de ruído mínimo (MNF). Todos os resultados foram analisados pela programação do software livre $R$, estes resultados experimentais indicaram claramente que o método MNF é o mais adequado para detectar o derramamento de óleo com maior precisão através de imagens multiespectrais Landsat 8.

Palavras-chave: Landsat 8 , derramamento de óleo, Repsol, normalização, processamento de imagens, ACP e MNF. 


\section{Introducción}

En la madrugada del 15 de enero de 2022 un derrame de petróleo causado por el buque de bandera italiana Mare Doricum, propiedad de la empresa española Repsol, en los mares del distrito de Ventanilla contaminó la costa central del Perú. Información inicial indicaba que el derrame había ocurrido en Ventanilla (en la provincia constitucional del Callao) durante las operaciones de descarga que se realizaban en la refinería La Pampilla. El Ministerio del Ambiente de Perú informó que al menos 11900 barriles de petróleo fueron derramados en el mar, lo cual condujo al peor desastre ecológico de la historia del país ocasionando un grave perjuicio no solo a la flora y fauna marina sino también a cientos de familias de pescadores peruanos quedando sin trabajo. Por su parte, la empresa Repsol, operadora de la Refinería La Pampilla, informó que unos 10396 barriles podrían haberse derramado el 15 de enero. A nivel mundial, existe una clasificación para la magnitud de derrames de petróleo; así, se tienen aquellos catalogados como derrames pequeños (menores a 7 toneladas), derrames medianos (entre 7 y 700 toneladas) y derrames de gran magnitud (mayores a 700 toneladas) (ITOPF, 2020). Lo ocurrido en Ventanilla se cataloga como un derrame de gran magnitud (equivalente a 1862 toneladas de petróleo aproximadamente). Los derrames de petróleo pueden ocurrir a partir de grietas en los oleoductos y plataformas de perforación de petróleo. En las zonas costeras, los derrames de petróleo ocurren principalmente debido a descargas ilegales e incidentes con barcos. La dispersión del derrame de petróleo en las áreas costeras debe ser monitoreada antes y después de este evento para asegurar que la costa esté a salvo de contaminantes.

Se han desarrollado varias técnicas para monitorear los derrames de petróleo, como sondeos visuales con aeronaves, radiómetros de microondas (MWR), escáner de línea infrarroja (IR), sensor de fluoro láser (LFS), radar aerotransportado lateral (SLAR), radar de apertura sintética (SAR), y sensores ópticos (como Landsat, Sentinel 2, Advance Spaceborne Thermal Emission and Reflection Radiometer (ASTER)) (Misra y Balaji 2017; Montali et al. 2006; Solberg et al. 2007; Trivero et al. 2007; Gade 2015; Gade et al. 1998, 2013; Shuchman et al. 2004; Richards 1999). A partir de estas técnicas, los sensores satelitales de teledetección que trabajan con diferentes anchos de banda en el espectro electromagnético, como el visible, el infrarrojo cercano, el infrarrojo de onda corta, el infrarrojo térmico (sensores ópticos) y el radar (SAR), se pueden usar de manera más eficiente debido al monitoreo continuo y amplia cobertura global de la Tierra. 
Dado que las imágenes multiespectrales son convenientes y baratas para detectar derrames de petróleo, se han propuesto muchos métodos basados en imágenes multiespectrales. Arslan, N. (2018) evaluó derrames de petróleo utilizando sensores multiespectrales Landsat 8 y se aplicó diferentes técnicas de procesamiento de imágenes a las bandas Landsat 8, como la fracción mínima de ruido (MNF), la morfología y los filtros de convolución para resaltar el área del derrame de petróleo en la costa de la bahía de Ildir (Izmir, Turquía). Bayramov et al. (2018) utilizó los sensores satelitales Sentinel1, Landsat-8, Radarsat, Envisat y ERS para monitorear derrames de petróleo en el Mar Caspio. Del mismo modo, Pashayev et al. (2018) rastrearon derrames de petróleo de petróleo en el Mar Caspio. Yu Mo, Michael S. Kearney and J. C. Alexis Riter (2017) desarrollaron un método para el monitoreo de derrames de petróleo de Deepwater Horizon en las marismas saladas de Luisiana utilizando imágenes Landsat. Min-Sun Lee et al. (2016) lograron detectar derrames de petróleo en la bahía de Gwangyang en la costa sur de Corea a partir de los datos OLI de DubaiSat-2 y Landsat 8 utilizando canales VIS/NIR con alta resolución espacial y clasificaron los derrames de petróleo en dos tipos, como petróleo espeso y petróleo similar a una película. Bahaa Mohamadi, Zhong Xie and Fujiang Liu (2015), monitorearon derrames de petróleo en el delta del Níger con imágenes Landsat 7 y Landsat 8 utilizando un modelo de evaluación de riesgos de derrames de petróleo basado en el Sistema de Información Geográfica. Qianguo Xing (2015) demostró que las imágenes TIR de Landsat 8 también podrían usarse para detectar derrames de petróleo. Dominique Dubucq (2016) descubrió que las imágenes de datos multiespectrales en el infrarrojo cercano (NIR) y el infrarrojo de onda corta (SWIR) eran competentes para la detección de derrames de petróleo.

La principal motivación en este estudio es desarrollar una detección basado en datos Landsat 8 para que la ubicación del derrame de petróleo pueda monitorearse con alta precisión. Esta investigación propuso la Fracción mínima de ruido (MNF sus siglas en inglés) después de haber realizado un preprocesamiento de imágenes a nivel de reflectancia y normalización a los datos con el fin de detectar la región del derrame de petróleo. Se analizó el área afectada utilizando la imagen de Landsat 8 descargada de la página oficial de la USGS. Todos los análisis fueron procesados en el software libre R.

\section{2. Área de estudio}

En esta investigación se utilizó la imagen satelital Landsat 8 Nivel 1 descargada de la página web oficial de la USGS el 01 de febrero del 2022 de la zona afectada por el 
derrame de petróleo en la costa del Perú (en las playas de Tomaycalla y Pasamayo hasta la comunidad ecológica Eco Truly Park). Los datos originales contienen 11 bandas. Después de la corrección atmosférica, se mantuvieron 8 bandas (Costera - Aerosoles, azul, verde, rojo, Infrarrojo cercano, Infrarrojo de onda corta 1, Infrarrojo de onda corta 2 y Cirrus) las cuales fueron utilizadas convenientemente en este estudio dado que tienen $30 \mathrm{~m}$ de resolución espacial en un rango de $0.435 \mu \mathrm{m}$ a $2.294 \mu \mathrm{m}$ en las imágenes de reflectancia. Los datos de reflectancia fueron los datos de entrada legales de esta investigación.

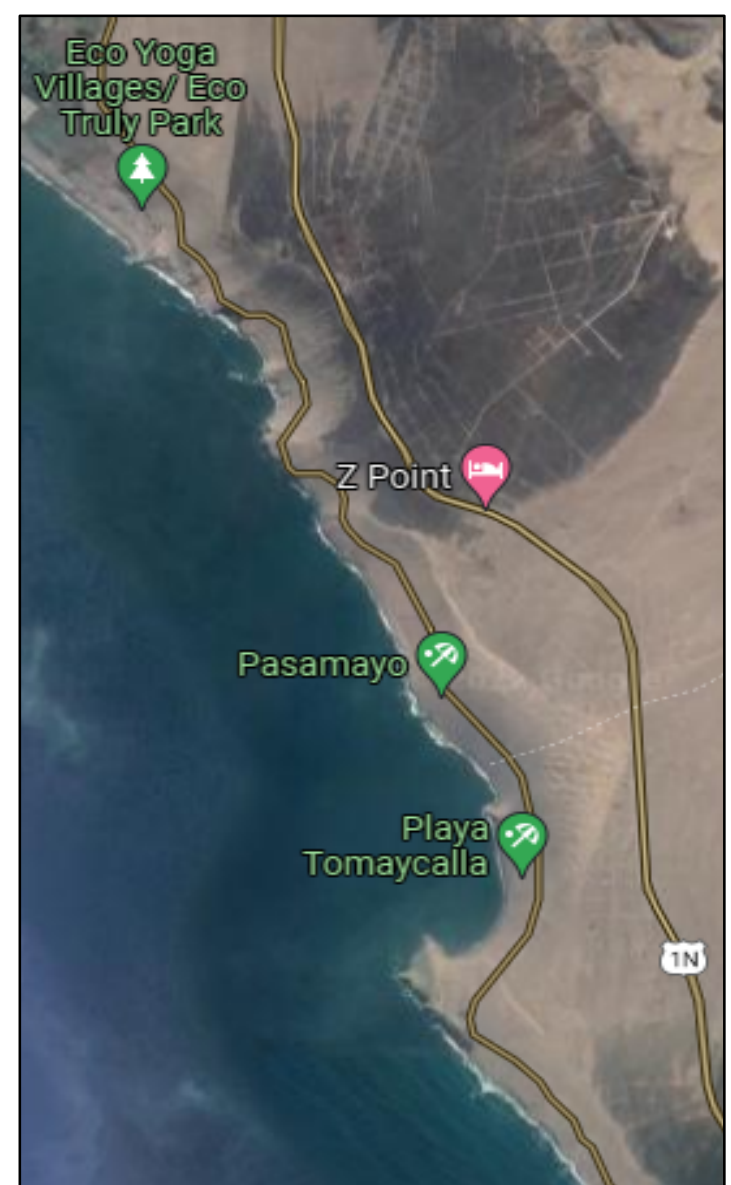

Figura 1. Zona de estudio visualizada en Google Maps.

\section{Metodología de trabajo}

Primeramente, se descargó la imagen satelital Landsat 8 colección 2 nivel 1 (sensor OLI) en la zona afectada por el derrame de petróleo en la página oficial de la USGS. Se procedió a convertir la imagen a reflectancia, luego se utilizó la normalización de Bandmax-min y finalmente de utilizaron los métodos de Análisis de Componentes 
Principales (ACP) y Fracción Mínima de Ruido (MNF por sus siglas en inglés) para poder detectar el derrame de petróleo en la zona afectada.

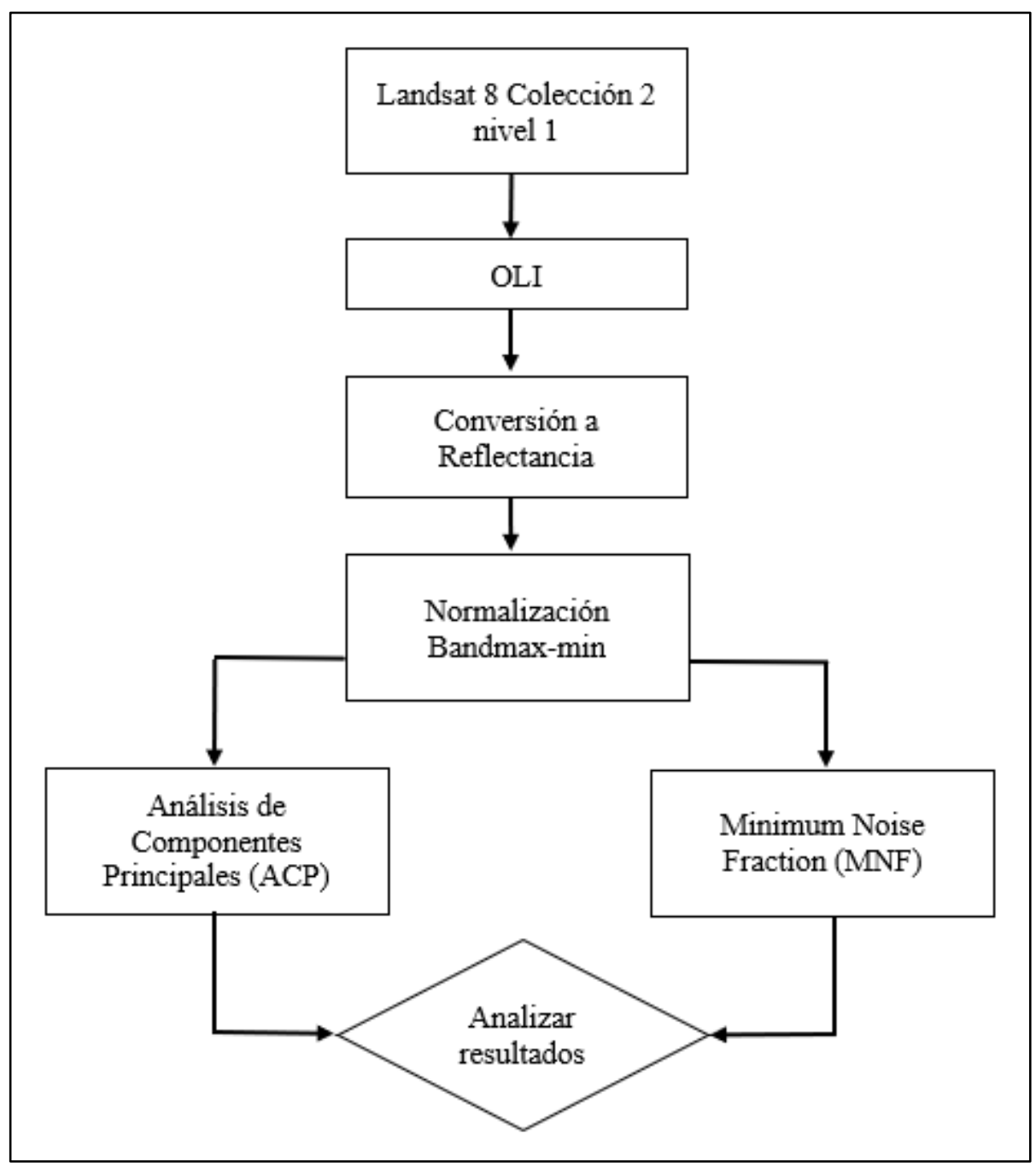

Figura 2. Flujo de trabajo utilizado en el estudio.

\section{Procesamiento de datos Landsat 8}

\section{Conversión de la imagen a Reflectancia con la Corrección DOS1}

El Dark Object Subtraction (DOS) o substracción de objeto oscuro es una familia de correcciones atmosféricas basadas en imagen. Chavez (1996) explica que "la suposición básica es que en la imagen algunos píxeles están completamente en sombra, y sus radiancias recibidas en el satélite se deben a la dispersión atmosférica (efecto bruma). Esta suposición es combinada con el hecho de que muy pocos elementos en la superficie de la Tierra tienen un color negro absoluto, por lo tanto, una reflectancia asumida de uno 
por ciento es mejor que un cero por ciento”. Es importante mencionar que la exactitud de las técnicas basadas en imagen es generalmente menor que las correcciones basadas en medidas físicas, pero son muy útiles cuando no están disponibles las mediciones atmosféricas que puedan mejorar la estimación de la reflectancia de la superficie terrestre. El efecto bruma es dado por (Sobrino, et al., 2004):

$$
L_{P}=L_{\min }-L_{D O 1 \%}
$$

donde:

$L_{\min }=$ "radiancia que corresponde a un valor digital para el cual la suma de todos los píxeles con valores digitales menores o iguales a este valor es igual al $0.01 \%$ de todos los píxeles de la imagen considerada" (Sobrino, et al., 2004, p. 437), por lo tanto, la radiancia obtenida con ese valor de conteo digital $\left(\mathrm{DN}_{\mathrm{min}}\right)$.

$L_{D O 1 \%}=$ radiancia del objeto oscuro, con un valor asumido de reflectancia de 0.01

En particular para imágenes Landsat se tiene que:

$$
L_{\text {min }}=M_{L} D N_{\min }+A_{L}
$$

Las imágenes landsat 8 son convertidas a radiancia antes del cálculo DOS1.

La Radiancia de Objeto Oscuro es dada por (Sobrino, et al., 2004):

$$
L_{D 01 \%}=0.01\left[\left(E S U N_{\lambda} \cos \theta_{s} T_{z}\right)+E_{\text {down }}\right] T_{v} /\left(\pi d^{2}\right)
$$

Por lo tanto, el efecto bruma es:

$$
L_{p}=M_{L} D N_{\min }+A_{L}-0.01\left[\left(E S U N_{\lambda} \cos \theta_{s} T_{z}\right)+E_{d o w n}\right] T_{v} /\left(\pi d^{2}\right)
$$

Existen varias técnicas DOS (ej. DOS1, DOS2, DOS3, DOS4), basadas en diferentes asunciones acerca de $T_{v}, T_{z}, \mathrm{y} E_{\text {down }}$. La técnica más simple es la DOS1, donde se hacen las siguientes suposiciones (Moran et al., 1992): $T_{v}=1, T_{z}=1$ y $E_{\text {down }}=0$. Por lo tanto, el efecto bruma es:

$$
L_{p}=M_{L} D N_{\min }+A_{L}-0.01 E S U N_{\lambda} \cos \theta_{s} /\left(\pi d^{2}\right)
$$

Y la reflectancia de la superficie terrestre resultante está dada por:

$$
\rho=\left[\pi\left(L_{\lambda}-L_{p}\right) d^{2}\right] /\left(E S U N_{\lambda} \cos \theta_{S}\right)
$$

Los valores ESUN $[W /(m 2 x \mu m)]$ para sensores Landsat 8 se encuentran en la tabla 1. 


\begin{tabular}{|c|c|c|c|c|c|c|c|c|c|}
\hline \multirow{2}{*}{$\begin{array}{c}\text { ECNO } \\
\text { HUMANISMO }\end{array}$} & \multicolumn{9}{|c|}{$\begin{array}{r}\text { Análisis de imágenes satelitales Landsat } 8 \text { por componentes principales y MNF para la detección de zonas del } \\
\text { derrame de petróleo Repsol en la Costa Peruana }\end{array}$} \\
\hline & & & & \multicolumn{6}{|c|}{ Jaime Yelsin Rosales Malpartida, Hebbert Alexander Apaza Mamani } \\
\hline Banda & 1 & 2 & 3 & 4 & 5 & 6 & 7 & 8 & 9 \\
\hline $\begin{array}{l}\text { Landsat } 8 \\
\text { OLI }\end{array}$ & 1895.33 & 2004.57 & 1820.75 & 1549.49 & 951.76 & 247.55 & 85.46 & 1723.88 & 366.97 \\
\hline
\end{tabular}

Tabla 1. Valores predetermiandos de las bandas landsat 8 OLI.

\section{Normalizacion de la imagen con el método Bandmax-min}

Para el preprocesamiento de datos de la imagen Landsat 8 después de tener la imagen convertida a reflectancia, realizamos el método de la normalización recomendada de Bandmax-min (Faxian Cao, et al., 2017).

$$
x_{i j}^{*}=\frac{x_{i j}-\operatorname{bandmin}\left(x_{i j}\right)}{\operatorname{bandmax}\left(x_{i j}\right)-\operatorname{bandmin}\left(x_{i j}\right)}
$$

\section{Análisis de componentes principales}

El análisis de componentes principales de datos de imágenes de teledetección se ha utilizado para varios propósitos de mapeo y extracción de información (Taylor, 1974; Fontanel et al., 1975; Blodget et al., 1978; Schowengerdt, 2012). Es una transformación matemática que genera nuevas imágenes, denominadas componentes o ejes, que son combinaciones lineales de las imágenes originales. ACP permite al usuario generar un nuevo conjunto de ejes rotados; estos ejes son ortogonales entre sí y las nuevas imágenes no tienen correlación matemática entre sí. La mayor cantidad de la varianza total se asigna al primer componente, y la varianza decreciente va a cada uno de los siguientes componentes. La suma de la varianza en todos los componentes es igual a la varianza total presente en las imágenes de entrada originales.

Uno de los principales usos de ACP es reducir la cantidad de imágenes o variables que se necesitan para el análisis, es decir, la reducción de la dimensionalidad. Por ejemplo, ACP se puede aplicar a las ocho bandas reflectantes de Landsat 8 y solo los tres primeros componentes se pueden usar para composición de color, análisis digital y/o clasificación. Estos primeros tres componentes tendrán un gran porcentaje de la variación total presente en las ocho bandas originales de Landsat 8. A menudo se desea la reducción de la dimensionalidad debido al gran volumen de datos que están presentes y las demandas computacionales o el límite de tres bandas en la composición de colores. Sin embargo, dos problemas que se pueden encontrar con los resultados de las imágenes ACP 
estándar cuando se usan de esta manera son (1) la información que no está asignada a los primeros tres componentes puede ser de gran interés, dependiendo del grado de correlación y contraste espectral que existen entre las ocho bandas Landsat 8 (Chávez et al., 1984; Williams, 1983); y (2) un compuesto de color hecho de tres de los ocho componentes puede ser difícil de interpretar visualmente (Williams, 1983).

\section{Fracción mínima de ruido}

El concepto matemático de fracción de mínimo ruido (MNF por sus siglas en inglés) depende del método de análisis de componentes principales (ACP). En el método MNF, la fracción de ruido se minimiza recursivamente mediante el uso de los mismos valores y vectores propios obtenidos del ACP, lo que da como resultado bandas de salida no correlacionadas (Green et al. 1988; Berman et al. 2012). La mayor parte de la información se puede encontrar en las primeras bandas de salida. Los datos comprenden $d$ dimensiones y $n$ píxeles. Sea $\mathrm{X}$ el tamaño de la matriz de datos $n \times d$ con el tamaño del vector $d \times 1$ de $X_{i}, i=1,2,3, \ldots, n$ :

$$
X=\left(X_{1}, \ldots, X_{n}\right)^{T}
$$

$X_{i}$ se puede obtener de la suma de la señal $\left(S_{i}\right)$ y el ruido $\left(\varepsilon_{i}\right)$ como:

$$
X_{i}=S_{i}+\varepsilon_{i}(10)
$$

Si no hay correlación entre la señal y el ruido, la covarianza de $X_{i}$ se puede representar de la siguiente manera:

$$
\Sigma_{\mathrm{X}}^{\mathrm{T}}=\Sigma_{\mathrm{X}}^{\mathrm{S}}+\Sigma_{\mathrm{X}}^{\mathrm{N}}
$$

donde $\Sigma_{\mathrm{X}}^{\mathrm{T}}$ es la covarianza de $X_{i}, \Sigma_{\mathrm{X}}^{\mathrm{S}}$ es la covarianza de $S_{i}$, y $\Sigma_{\mathrm{X}}^{\mathrm{N}}$ es la covarianza de $\varepsilon_{i}$. Para cálculos matemáticos, las matrices de covarianza de la señal y el ruido se diagonalizan utilizando la matriz $\mathrm{A}$ con dimensión $\mathrm{d} \times \mathrm{d}$ que incluye los vectores propios de los datos (ecuación 12).

$$
\mathrm{A} \Sigma_{\mathrm{X}}^{\mathrm{S}} \mathrm{A}^{\mathrm{T}}=\Lambda \mathrm{A} \Sigma_{\mathrm{X}}^{\mathrm{N}} \mathrm{A}^{\mathrm{T}}=\mathrm{I}
$$

En esta ecuación, $\Lambda$ es una matriz diagonal de valores propios con $\lambda_{1} \geq \lambda_{2} \geq \cdots \geq$ $\lambda_{d} \geq 0$ e I es la matriz identidad. Para resolver la ecuación 12, el método de descomposición propia generalizada se puede utilizar para transformaciones (Green et al. 1988; Berman et al. 2011). 


\section{Resultados y discusiones}

Para detectar el derrame de petróleo, las imágenes de Landsat 8 descargadas se procesaron con la metodología dada. Para comparar las mediciones ópticas in situ con la señal del satélite, las radiancias medidas debían convertirse en reflectancias definida en la ecuación (6) y se puede apreciar en RGB en la figura 3.

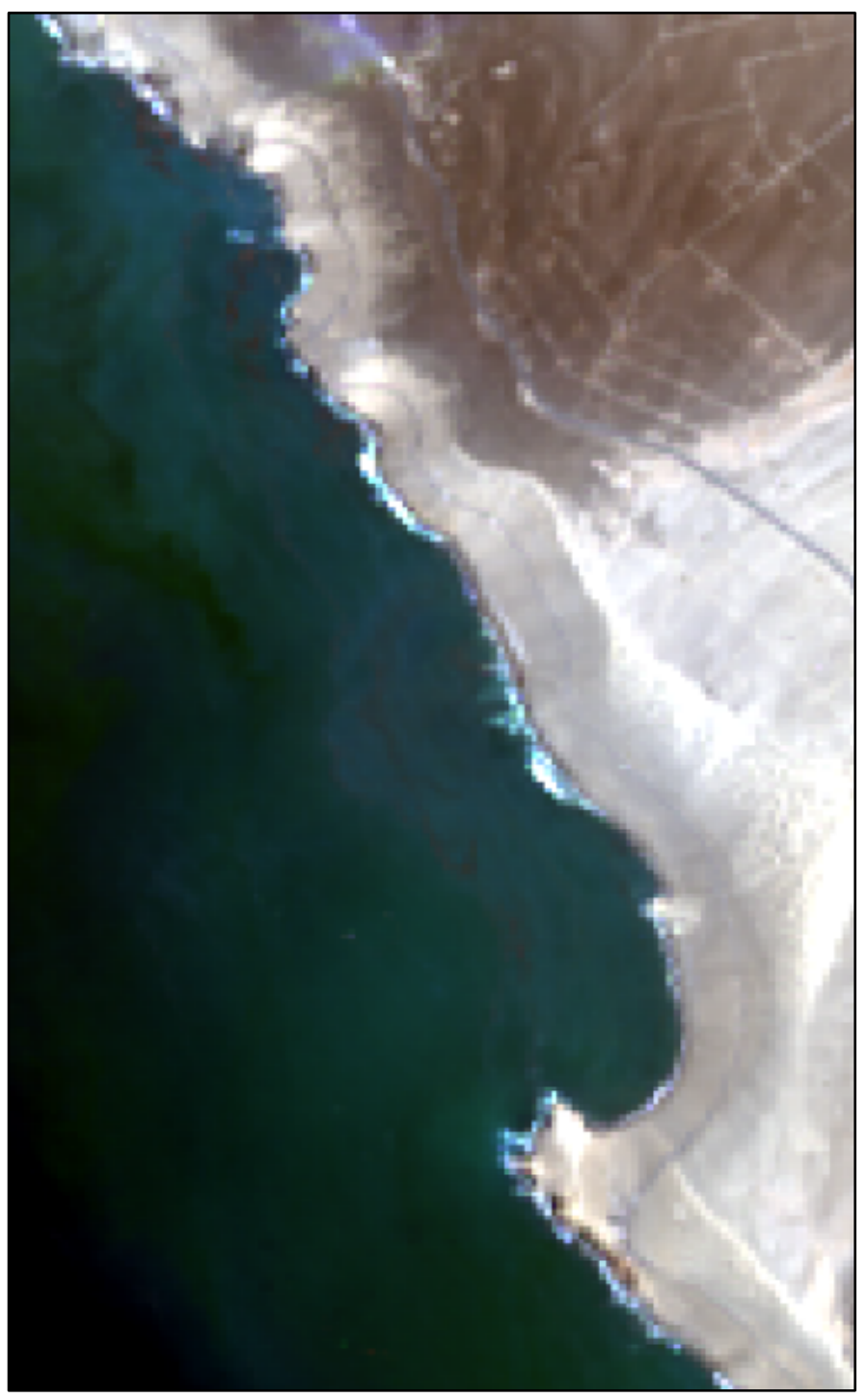

Figura 3. Imagen convertida a reflectancia en la zona del derrame de petróleo.

Para tener una mejor visualización del derrame de petróleo de procedió a mostrar dicha conversión en el falso color utilizando las bandas 4,5 y 7 y se puede apreciar en la flecha verde en la figura 4. 


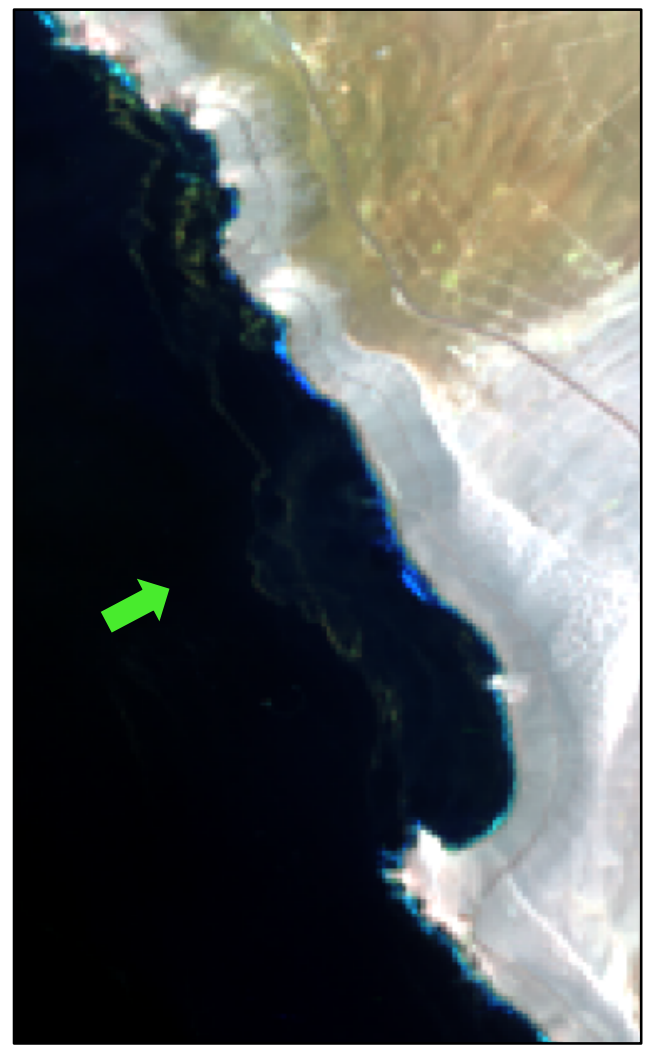

Figura 4. zona del derrame de petróleo: imagen convertida a reflectancia mostrado con el falso color.

Una vez realizado la conversión en reflectancia se aplicó la normalización con el método recomendado de Bandmax-min la cual nos permite separar mejor en grupos los elementos que se forman de banda a banda, por ejemplo, la banda 3 vs banda 5 después de aplicar la normalización por el método Bandmax-min podemos ver cómo se separan en dos grupos los elementos formados de banda a banda y se muestra en la figura 5. 


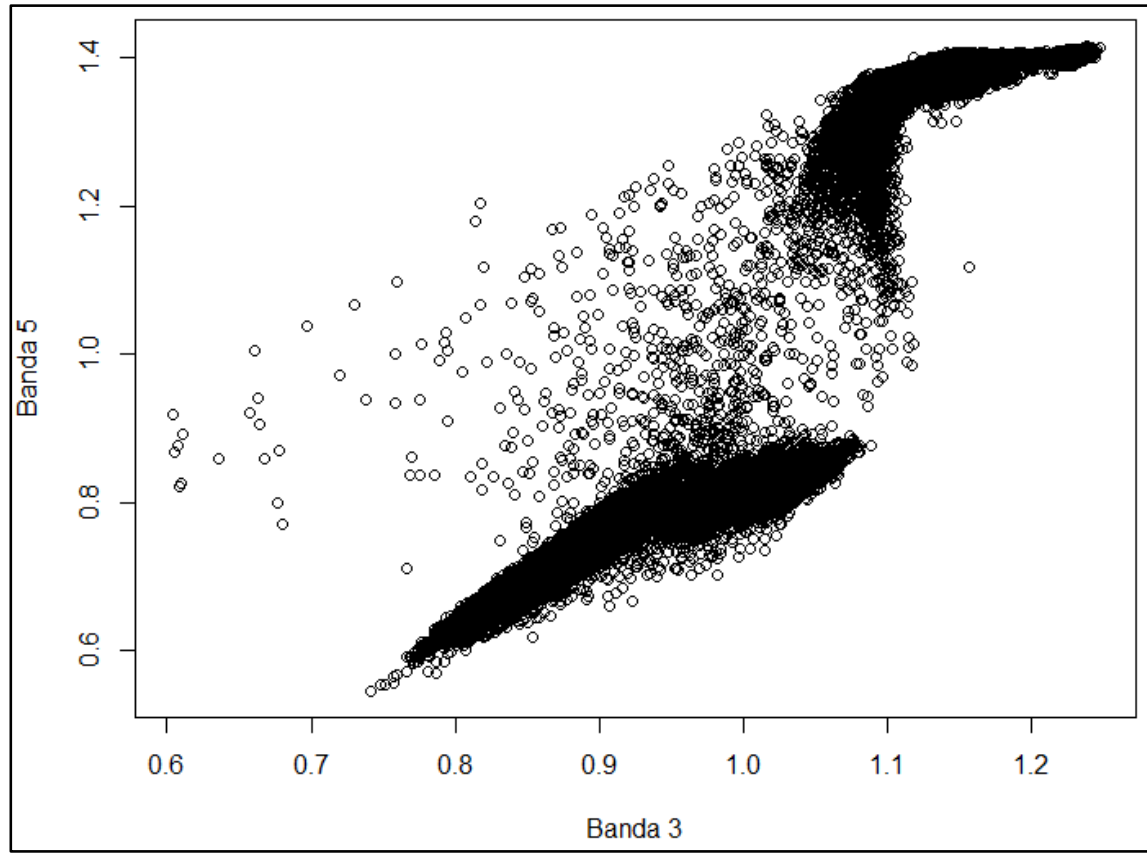

Figura 5. Gráfico de la banda 3 (verde) vs banda 5 (infrarrojo cercano).

Después de la normalizar los datos procedemos aplicar los métodos de análisis de componentes principales (ACP) y la Fracción mínima de ruido (MNF) a las 8 bandas espectrales de la imagen Landsat 8 de resolución espacial de 30 metros (Costera Aerosoles, azul, verde, rojo, Infrarrojo cercano, Infrarrojo de onda corta 1, Infrarrojo de onda corta 2 y Cirrus) para mostrar la contribución de estas bandas en los incidentes de derrames de petróleo. Estos métodos se pueden utilizar para distinguir el área con derrame de petróleo que tiene un contraste diferente de las áreas circundantes. Se seleccionaron ocho bandas de la imagen Landsat 8 como entrada, lo que resultó en ocho bandas de salida después del procesamiento. Los resultados al aplicar ACP se muestran en la tabla 2 con la importancia de cada componente según la varianza y en la figura 6 se muestra los componentes principales de la zona afectada.

\begin{tabular}{|l|l|l|l|l|l|l|l|l|}
\cline { 2 - 9 } \multicolumn{1}{c|}{} & PC1 & PC2 & PC3 & PC4 & PC5 & PC6 & PC7 & PC8 \\
\hline Desviación estándar & 2.564 & 0.977 & 0.669 & & 0.065 & 0.049 & 0.032 & 0.018 \\
Proporción de & 9 & 9 & 0.1093 & 4 & 1 & 4 & 9 \\
varianza & 0.821 & 0.119 & 0.056 & & 0.000 & 0.000 & 0.000 & 0.000 \\
\hline $\begin{array}{l}\text { Proporción } \\
\text { acumulada }\end{array}$ & 9 & 5 & 1 & 0.0015 & 5 & 3 & 1 & 0 \\
\hline
\end{tabular}

Tabla 2. Resumen del analisis de ACP. 


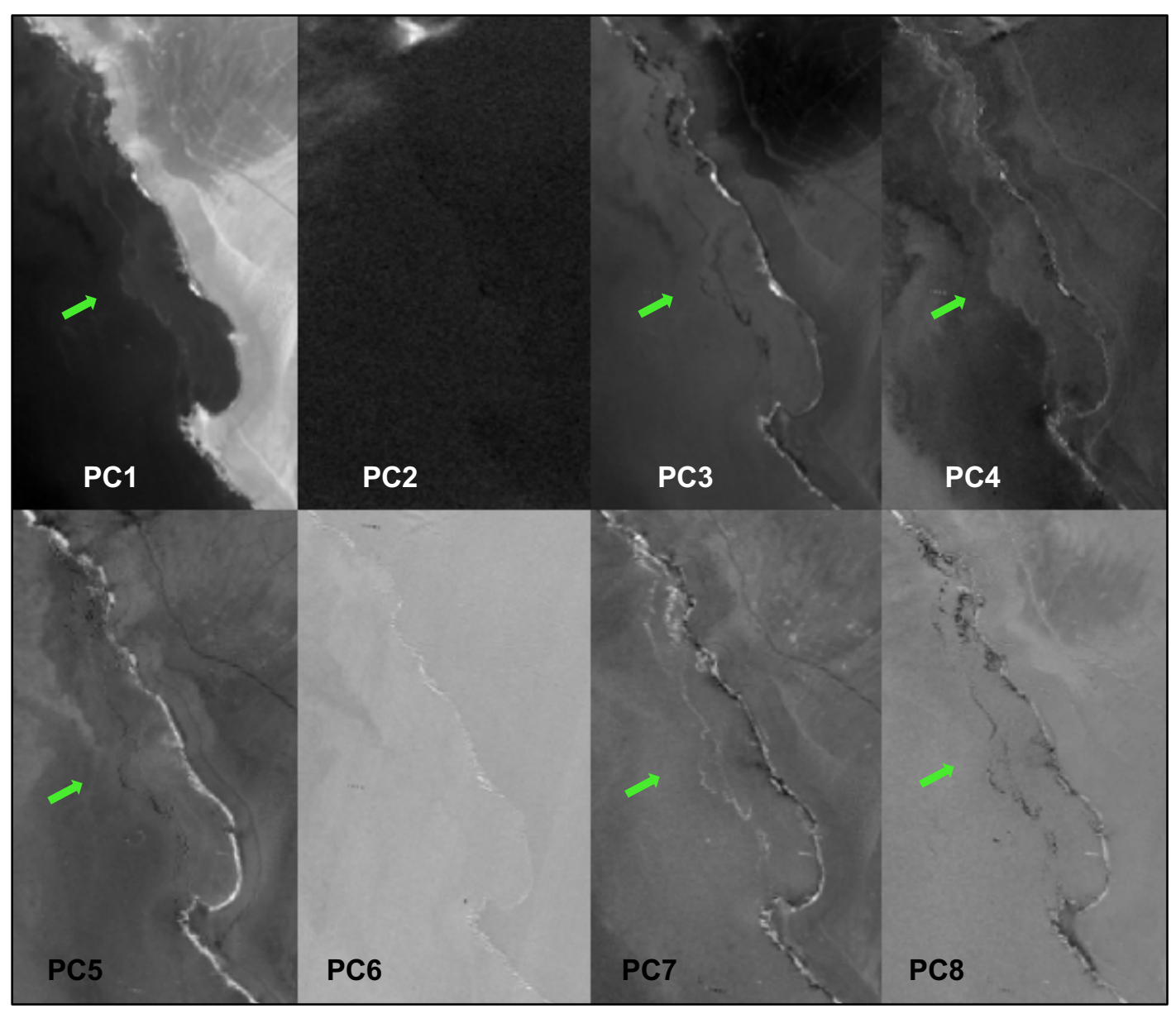

Figura 6. Componentes principales aplicado a la zona afectada por el derrame de petróleo: la primera componente se muestra como PC1, la segunda como PC2 así sucesivamente hasta la componente PC8 y se aprecia en flecha verde la línea el derrame de petróleo.

De la figura 6 y la tabla 2 podemos ver que a pesar que la primera componente (PC1) capta el $82.19 \%$ de la variabilidad de información se puede apreciar el derrame de petróleo con cierta dificultad. Por otra parte, con las componentes principales PC3, PC4, PC5, PC7 y PC8 se logra visualizar el derrame de petróleo con cierto ruido de la imagen por lo que no se logra distinguir bien el derrame de petróleo con el agua del mar y por último las componentes PC2 y PC6 no distingue casi nada el derrame de petróleo debido también al ruido de la imagen. 


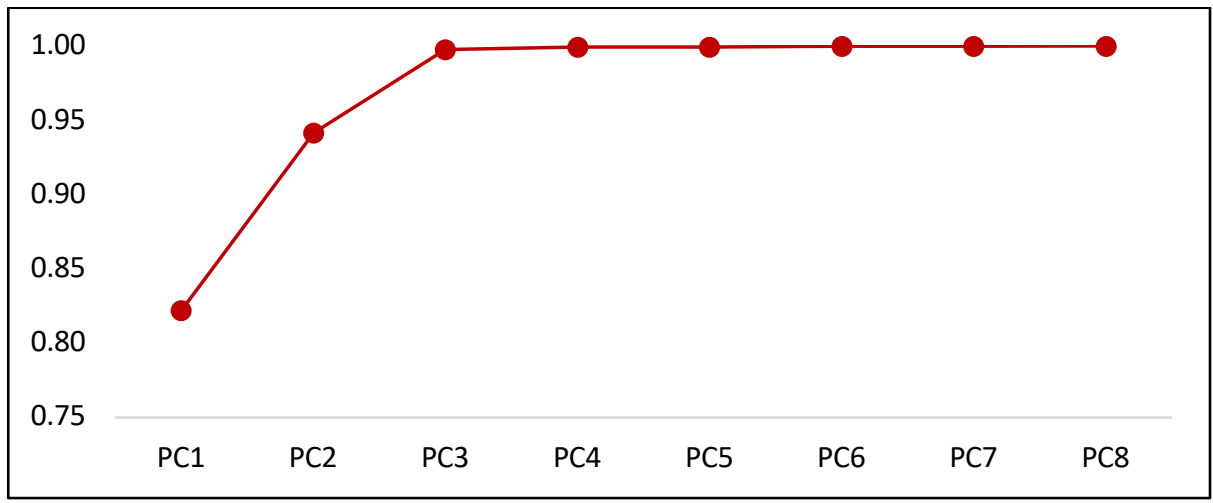

Figura 7. Proporción acumulada de varianza de los componentes principales.

De la figura 7 podemos ver que los tres primeros componentes logran capturar la máxima variación de la información de las bandas, sin embargo, no es suficiente para detectar con precisión al derrame del petróleo.

Los resultados al aplicar el método MNF se muestran en la figura 8 y 9 donde se muestra claramente cómo el primer valor propio separa de manera más precisa respecto a los demás valores propios el ruido de la imagen del área del derrame de petróleo en la zona afectada y se muestra con flecha verde el primer valor propio en la figura 9 (VP1), es por esta razón que se logra distinguir el derrame de petróleo del mar con una línea en gris, mientras que los demás valores propios no separan casi nada dicho ruido, por ello no se puede distinguir bien el derrame de petróleo y se aprecia también en la figura 8 en la relación señal-ruido. De esta manera obtenemos una mejor detección del derrame de petróleo con el método MNF la cual podemos visualizar de manera más clara en la figura 10 donde graficamos de otra manera el primer valor propio con la función image en $\mathrm{R}$ utilizando el parámetro color de dos distintas maneras.

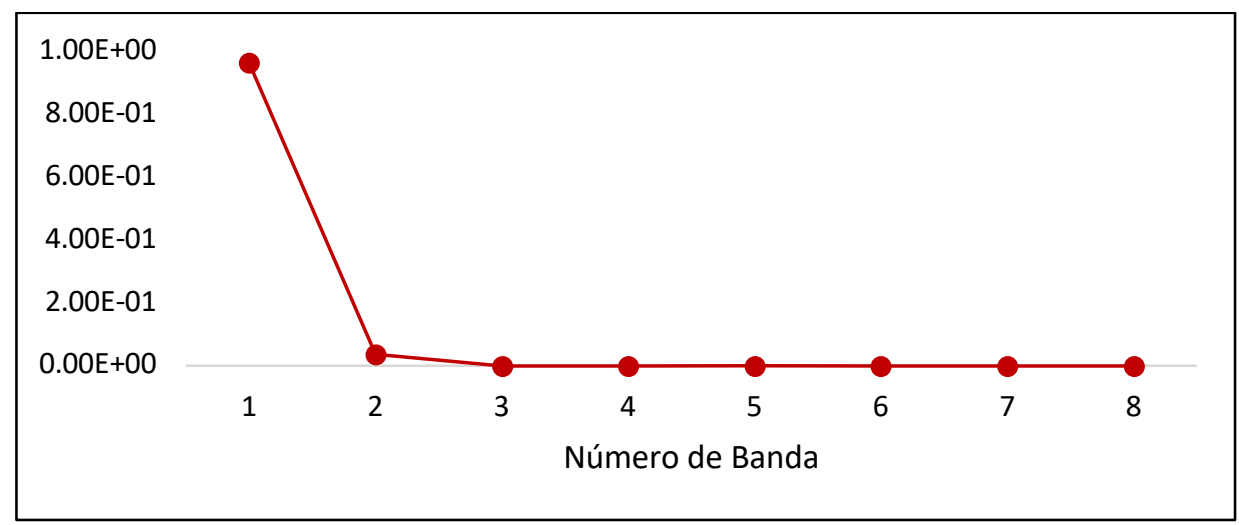

Figura 8. Relación señal-ruido de las 8 bandas procesadas. 


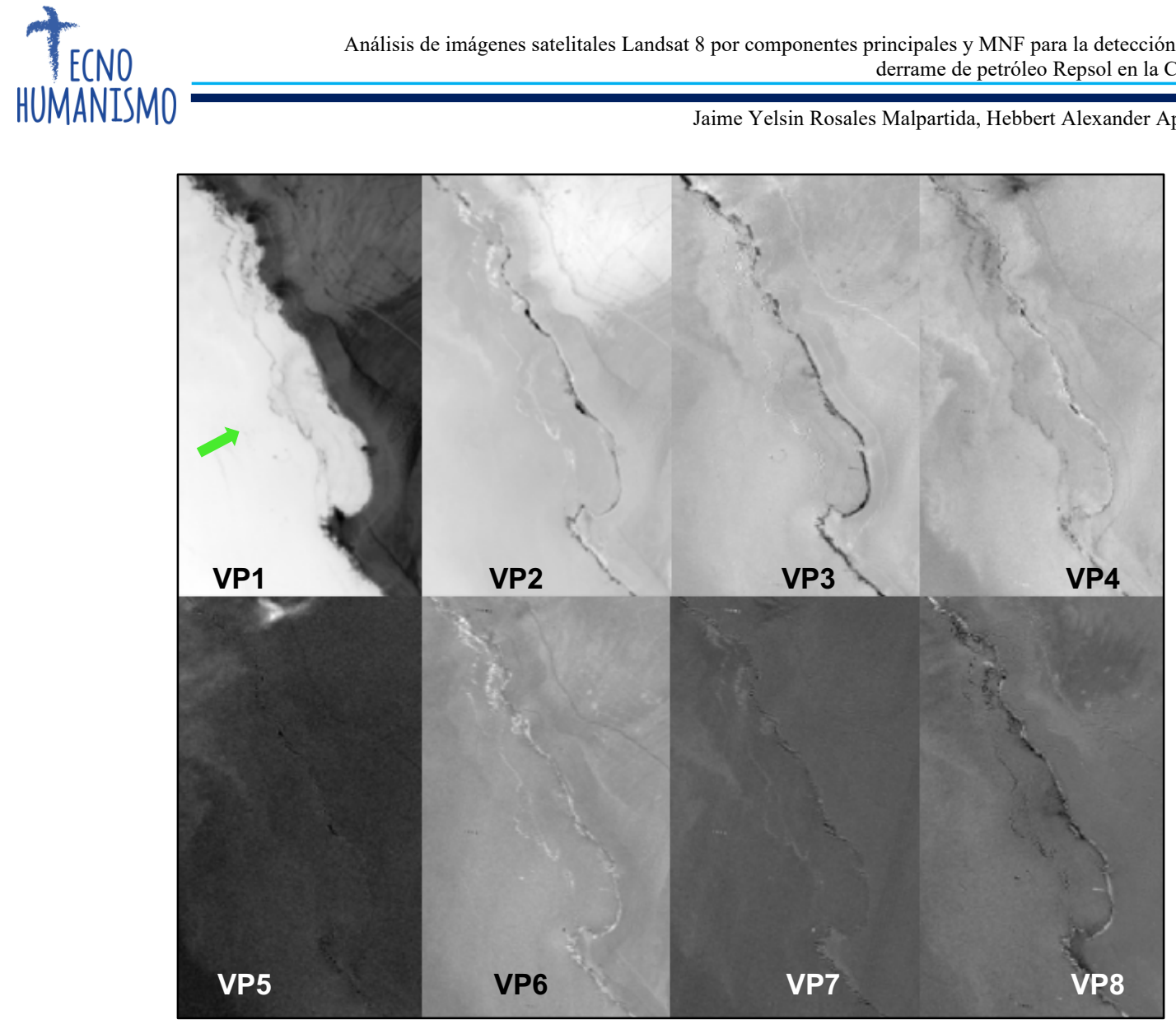

Figura 9. Aplicación del MNF a la zona afectada por el derrame de petróleo: el primer valor propio se muestra como VP1, la segunda como VP2 así sucesivamente hasta el valor propio VP8 y se aprecia en flecha verde la línea el derrame de petróleo.

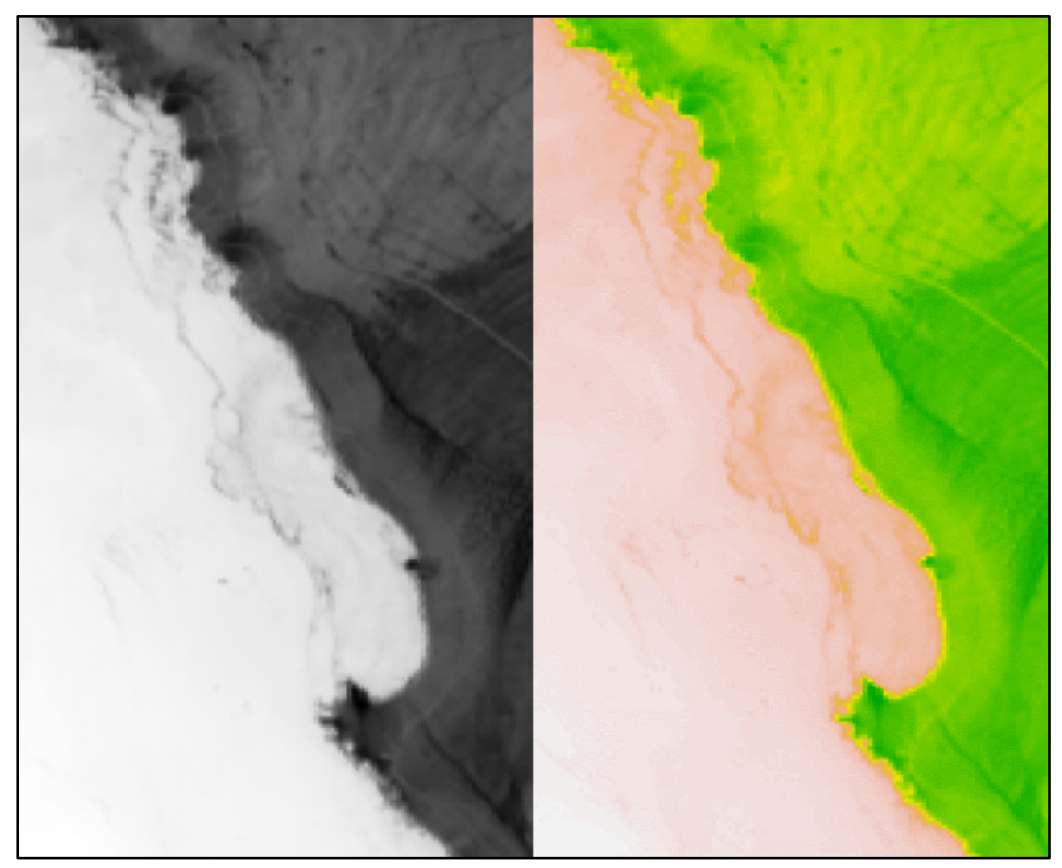

Figura 10. Visualización del derrame de petróleo en $\mathrm{R}$ con el primer valor propio del método MNF: (a) con la función image y el parámetro col = gray $((0: 256) / 256)$, (b) con la función image y el parámetro col=terrain.colors(100). 


\section{Conclusiones}

Los resultados experimentales indicaron que el método MNF es adecuado para detectar el derrame de petróleo en la costa peruana (en las playas de Tomaycalla y Pasamayo hasta la comunidad ecológica Eco Truly Park) utilizando imágenes multiespectrales de Landsat 8, dado que este método separa el ruido de la imagen logrando detectar de forma precisa el derrame de petróleo ocurrido en la costa peruana causado por la empresa Repsol con el primer valor propio del método MNF, Sin embargo, con el método de ACP se puede apreciar la zona del derrame de petróleo con más dificultad debido a que este método no separa el ruido de la imagen, por lo tanto no permite diferenciar de manera clara el petróleo del agua del mar de la costa peruana, por lo tanto, se recomienda utilizar el método MNF para este estudio.

\section{Referencias}

Arslan, N. (2018). Assessment of oil spills using Sentinel 1 C-band SAR and Landsat 8 multispectral sensors. Environmental monitoring and assessment, 190(11), 1-14.

Bayramov, E., Kada, M., \& Buchroithner, M. (2018). Monitoring oil spill hotspots, contamination probability modelling and assessment of coastal impacts in the Caspian Sea using SENTINEL-1, LANDSAT-8, RADARSAT, ENVISAT and ERS satellite sensors. Journal of Operational Oceanography, 11(1), 27-43.

Berman, M., Phatak, A., \& Traylen, A. (2012). Some invariance properties of the minimum noise fraction transform. Chemometrics and Intelligent Laboratory Systems, 117, 189-199.

Cao, F., Yang, Z., Ren, J., Jiang, M., \& Ling, W. K. (2017). Does normalization methods play a role for hyperspectral image classification? arXiv preprint arXiv:1710.02939.

Chavez Jr, P. S. (1984). Image Processing techniques for Thermatic Mapper data. Proceedings, ASPRS-ACSM Technical Papers, 2, 728-742.

Chavez, P. S. (1996). Image-Based Atmospheric Corrections - Revisited and Improved. https://pdfs.semanticscholar.org/45f1/2625ce130261c7d360d50e09c635355ca919. pdf 
Dubucq, D., Sicot, G., Lennon, M., \& Miegebielle, V. (2016). Detection and discrimination of the thick oil patches on the sea surface. International Archives of the Photogrammetry, Remote Sensing and Spatial Information Sciences, 8.

Fontanel, A., Blanchet, C., \& Lallemand, C. (1975, June). Enhancement of Landsat imagery by combination of multispectral classification and principal component analysis. In NASA. Lyndon B. Johnson Space Center NASA Earth Resources Surv. Symp., Vol. 1-B (No. G-24).

Gade, M. (2015). Synthetic aperture radar applications in coastal waters. Proceedings of the twelfth international conference on the Mediterranean coastal environment MEDCOAST 06-10 October, Varna, Bulgaria.

Gade, M., Alpers, W., Huhnerfuss, H.,Wismann, V. R., \& Lange, P. A. (1998). On the reduction of the radar backscatter by oceanic surface films: scatterometer measurements and their theoretical interpretation. Remote Sensing of Environment, 66(1), 52-70.

Gade, M., Byfield, V., Ermakov, S., Lavrova, O., \& Mitnik, L. (2013). Slicks as indicators for marine processes. Oceanography, 26(2), 138-149.

Green, A. A., Berman, M., Switzer, P., \& Craig, M. D. (1988). A transformation for ordering multispectral data in terms of image quality with implications for noise removal. IEEE Transactions on geoscience and remote sensing, 26(1), 65-74.

ITOPF. (2020). Oil Tanker Spill Statistics 2020.

Lee, M. S., Park, K. A., Lee, H. R., Park, J. J., Kang, C. K., \& Lee, M. (2016, July). Detection and dispersion of oil spills from satellite optical images in a coastal bay. In 2016 IEEE International Geoscience and Remote Sensing Symposium (IGARSS) (pp. 2491-2494). IEEE.

Misra, A., \& Balaji, R. (2017). Simple approaches to oil spill detection using sentinel application platform (SNAP)-ocean application tools and texture analysis: a comparative study. Journal of the Indian Society of Remote Sensing, 45, 10651075. https://doi.org/10.1007/s12524-016-0658

Mo, Y., Kearney, M. S., \& Riter, J. C. (2017). Post-deepwater horizon oil spill monitoring of Louisiana salt marshes using landsat imagery. Remote Sensing, 9(6), 547. 
Mohamadi, B., Xie, Z., \& Liu, F. (2015). GIS based oil spill risk assessment model for the Niger Delta's vegetation. Nature Environment and Pollution Technology, 14(3), 545 .

Montali, A., Giacinto, G., Migliaccio, M., \& Gambardella, A. (2006). Supervised pattern classification techniques for oil spill classification in SAR images: preliminary results. Proceedings of the SEASAR2006 workshop, ESA-ESRIN, Frascati, Italy.

Moran, M. S., Jackson, R. D., Slater, P. N., \& Teillet, P. M. (1992). Evaluation of simplified procedures for retrieval of land surface reflectance factors from satellite sensor output. Remote Sensing of Environment, 41(2-3), 169-184. https://doi.org/10.1016/0034- 4257(92)90076-V

Pashayev, N.M., Ragimov, R.M., Samedov, F.R., Gahramanova, D.S., 2018. Aerospace monitoring of the oil pollution of the Caspian Sea on the base of GIS technology and radar space images. IFAC-PapersOnLine 51 (30), 558-560.

Richards, J. A. (1999). Remote sensing digital image analysis: an introduction. Berlin: Springer-Verlag.

Schowengerdt, R. A. (2012). Techniques for image processing and classifications in remote sensing. Academic Press.

Shuchman, R. A., Onstott, R. G., Johannessen, O. M., Sandven, S., \& Johannessen, J. A. (2004). Processes at the ice edge: the arctic. Synthetic Aperture Radar marine user's manual, 373-395.

Sobrino, J. A., Jiménez-Muñoz, J. C. and Paolini, L. 2004. 84 Land surface temperature retrieval from LANDSAT TM 85 5, Remote Sens. Environ., vol. 90, 434-44

Solberg, A., Brekke, C., \& Husoy, P. (2007). Oil spill detection in Radarsat and ENVISAT SAR images. IEEE Transactions on Geoscience and Remote Sensing, 45(3), 746-755.

Taylor, M. M. (1974, January). Principal components colour display of ERTS imagery. In NASA. Goddard Space Flight Center 3d ERTS-1 Symp., Vol. 1, Sect. B (No. PAPER-I12).

Trivero, P., Biamino, W, \& Nirchio, F. (2007). High resolution COSMO-SkyMed SAR images for oil spills automatic detection. Proceedings of the geoscience and remote sensing symposium, IGARSS 2007 IEEE International: 2-5. 
Williams, D. L., Irons, J. R., Markham, B. L., Nelson, R. F., Toll, D. L., Latty, R. S., \& Stauffer, M. L. (1983, August). Impact of TM sensor characteristics on classification accuracy. In Proc. IGARSS (Vol. 83, pp. 5-1).

Xing, Q., Li, L., Lou, M., Bing, L., Zhao, R., \& Li, Z. (2015). Observation of oil spills through landsat thermal infrared imagery: A case of deepwater horizon. Aquatic Procedia, 3, 151-156. 